\section{Tratamento anticoagulante de longa duração}

0 tratamento anticoagulante de longa duração tem como principal objetivo impedir a recorrência de TEV e a ocorrência de TEP fatal. Na decisão de manter o anticoagulante, é importante também estimar o risco de sangramento, pesando-se a relação risco-beneficio dessa terapia. 0 tempo de anticoagulação deve ser sempre individualizado diante do contexto do paciente. Entretanto, recomendações gerais baseadas nos ensaios clínicos e meta-análises podem ser feitas. ${ }^{(1)}$ Ressalta-se que os pacientes incluídos nos estudos são aqueles com TEV em geral, sendo a maioria com TVP e, excepcionalmente, TEP isolada. ${ }^{(2)}$ Do ponto de vista prático, não há diferenças no manejo, a longo prazo, de pacientes com TEP ou com TVP proximal, apesar de o risco de recorrência de TEV ser 3 vezes maior nos pacientes com TEP. ${ }^{(3)}$

A anticoagulação é realizada na maioria dos pacientes com o uso de AVK, sendo necessária à dosagem repetida de $\mathrm{RNI}$ a fim de manter o alvo de 2,5 (entre 2,0 e 3,0). Estudos antigos que tentaram utilizar HNF em minidoses mostraram que essa estratégia não foi eficaz em prevenir a recorrência da TEV quando comparada ao uso de AVK em dose plena. ${ }^{(4)}$ Mais recentemente, foi testado um alvo terapêutico mais baixo para os AVK (entre $1,5$ e 2,0$)$ que, comparado ao placebo, é eficaz em prevenir a recorrência da TEV, mas pior que a anticoagulação em níveis convencionais (alvo de 2,5). .5,6) Pacientes em tromboprofilaxia secundária com AVK devem manter o RNI entre 2,0 e 3,0. (A) Em situações especiais, onde há o desejo de monitorização menos frequente, pode-se optar por um alvo menos intenso de anticoagulação ao invés de suspender a anticoagulação.(B) As HBPM são alternativas tão eficazes quanto os $A V K$ na anticoagulação de longa duração e prescindem, na maioria dos casos, de monitorização laboratorial. São possivelmente mais seguras que os AVK e podem ser úteis, sobretudo, em pacientes que residem em áreas inacessiveis, que desejam menos consultas médicas para o controle de anticoagulação ou que tenham contraindicações aos AVK. ${ }^{(7,8)}$ As HBPM podem substituir os AVK na tromboprofilaxia secundária da TEV.(A) As questões de farmacoeconomia devem ser levadas em consideração e calculadas conforme os custos de aquisição das medicações no mercado.

A estimativa do risco de sangramento é fundamental na decisão do tempo do tratamento.
(D) ${ }^{(9)}$ Há fatores associados à maior frequência desse efeito adverso: idade avançada (sobretudo $>75$ anos), sangramento digestivo prévio (principalmente se causa não revertida), AVC prévio não cardioembólico, nefropatia, hepatopatia, uso concomitante de antiplaquetários, outras doenças graves, monitorização subótima e má adesão ao uso de anticoagulantes. Existem escores de predição de risco de sangramento que podem ser utilizados no contexto de TEV. ${ }^{(10)}$

Além da eficácia dos anticoagulantes e do inerente risco de sangramento, a definição da duração do seu uso depende da persistência dos fatores de risco. Um estudo em pacientes com TEP demonstrou uma taxa de recorrência de TEV de 2,5\% ao ano em pacientes com fatores de risco transitórios (por ex., cirurgia, doença clínica aguda, gestação, estrogenoterapia e trauma) e de 4,5\% ao ano em pacientes com fatores de risco permanentes ou não identificados. ${ }^{(2)}$ Ensaios clínicos em cenários com fatores de risco transitórios compararam a anticoagulação de curta duração (4-6 semanas) com a de duração intermediária (3-6 meses), demonstrando que o grupo de menor tempo de tratamento tem uma taxa elevada de recorrência. ${ }^{(11)}$ Portanto, em pacientes com fatores de risco transitórios, recomenda-se o tratamento anticoagulante por 3 meses. $(A)^{(12,13)}$

Estudos comparando tempos de anticoagulação intermediários (3, 6 ou 12 meses) não demonstraram diferenças significativas do ponto de vista prático. 0 risco de recorrência e sangramento não foi estatisticamente significativo em dois estudos que compararam 3 e 6 meses de uso de anticoagulantes em pacientes com o primeiro episódio de TEV. ${ }^{(13,14)}$ Em pacientes sem fatores de risco conhecidos (TEP não provocada ou idiopática), recomenda-se que o tratamento dure pelo menos 3 meses.(A) Após esse período, esses pacientes devem ser avaliados quanto aos riscos e benefícios de se manter a anticoagulação por tempo prolongado.(C) 0 benefício da anticoagulação permanece enquanto essa está em uso, não ocorrendo um efeito rebote de aumento da recorrência da TEV após a sua suspensão. Apesar do risco de novos episódios de TEV diminuírem ao longo do tempo, o risco de sangramento pela anticoagulação permanece independente do tempo de uso. ${ }^{(15,16)}$ Pacientes com primeiro episódio de TEP idiopática, baixo risco de complicações e boa adesão ao tratamento devem ser mantidos sob anticoagulação indefinidamente. $(A)^{(17)}$ Cabe ressaltar que, em situações nas quais a TEV foi descoberta aciden- 
talmente, a conduta terapêutica é a mesma. Em pacientes que apresentam recorrência de TEP idiopática (segundo episódio ou mais), recomenda-se o uso de anticoagulação indefinidamente.(A)

Pacientes com câncer têm um risco aumentado de recorrência de TEV, chegando a 20\% no primeiro ano após o evento inicial. ${ }^{(18)}$ Neoplasia maligna é o maior preditor de recorrência entre os fatores associados às características do paciente, sendo que a anticoagulação por tempo indefinido deve ser fortemente considerada. Nesses pacientes, o uso de HBPM mostrou-se mais eficaz que o de AVK na prevenção de novos episódios, mas sem modificação da sobrevida. ${ }^{(19,20)}$ Após 6 meses, não há estudos randomizados consistentes que favoreçam um dos anticoagulantes. Assim, recomenda-se o tratamento por 3-6 meses com HBPM em pacientes com câncer. (B) Após esse período, a anticoagulação deve ser mantida, com AVK ou HBPM, de forma indefinida ou até o câncer ser resolvido.(A)

Embora seja importante estratificar os pacientes com TEP não provocada (isto é, sem fatores de risco transitórios e sem câncer), esse é um tema complexo. Alguns contextos clínicos devem ser levados em consideração na decisão de se manter ou suspender a anticoagulação de longa duração, pois os seguintes contextos aumentam o risco de recorrência: TEV prévio (isto é, recorrente), sexo masculino, síndrome antifosfolipídeo, trombofilias hereditárias, trombose residual em veias próximas e presença de DVD verificada por ecocardiograma na alta hospitalar.(D) ${ }^{(9)}$

Pacientes portadores de trombofilias hereditárias de alto risco de recorrência, como homozigose para o fator $\mathrm{V}$ Leiden, homozigose para mutação do gene da protrombina ou dupla heterozigose para essas mutações, bem como a deficiência de proteína C, de proteína $\mathrm{S}$ ou de antitrombina, são candidatos à anticoagulação por tempo indefinido.(C) ${ }^{(21,22)}$ Nos casos de heterozigose isolada para o fator $\checkmark$ Leiden, de mutação do gene da protrombina ou hiper-homocisteinemia, os estudos não têm demonstrado benefícios da anticoagulação permanente, visto que o risco de recorrência não é tão elevado. ${ }^{(23)}$

Recentemente, foi lançado no mercado brasileiro o etexilato de dabigatrana, um inibidor reversível do sítio ativo da trombina. É um agente para uso oral que prescinde de monitorização laboratorial. A dose utilizada é de 150-220 mg/dia em uma tomada. Esse fármaco foi testado em ensaios clínicos de fase 111 na prevenção de TEV em cirurgias ortopédicas, sendo comparado a enoxaparina. Na maioria dos estudos, não houve diferença estatisticamente significativa nos desfechos de incidência de TEV, sangramento e mortalidade. ${ }^{(24-26)}$ É uma alternativa às HBPM na prevenção de TEV em pacientes ortopédicos.(B) Apesar de ter comprovada eficácia na anticoagulação plena no contexto da fibrilação atrial, ainda não foram liberados os resultados do uso da dabigatrana no tratamento de TEV. Seu uso nessa indicação ainda é incipiente, podendo ser discutido os riscos e benefícios com o paciente no contexto da trombocitopenia por heparina, já que no mercado brasileiro não há outras alternativas até o momento.(D)

Um recente estudo demonstrou que valores de dímeros $\mathrm{D}$ acima do plano de corte após a suspensão da anticoagulação têm sido associados a uma maior taxa de recorrência. ${ }^{(27)}$ Sugere-se a sua determinação cerca de 4 semanas após a descontinuação da anticoagulação, tendo em

Quadro 12 - Recomendações para a duração da tromboprofilaxia secundária. ${ }^{\mathrm{a}}$

\begin{tabular}{|ll|}
\hline \multicolumn{1}{|c|}{ Contexto } & \multicolumn{1}{c|}{ Tempo de anticoagulação recomendado } \\
\hline Primeiro episódio de TEV associado a fatores de risco transitórios & 3 meses \\
Primeiro episódio de TEV não provocada & Pelo menos 3 meses \\
& Se há baixo risco de sangramento, considerar \\
& anticoagulação de longa duração \\
Segundo episódio de TEV não provocada & A longo prazo \\
TEV associada a câncer & A longo prazo ou enquanto câncer ativo \\
TEV associada a trombofilias de alto risco & A longo prazo \\
TEV associada à heterozigose para fator V Leiden, heterozigose & Conforme contextos anteriores (essas \\
para mutação do gene da protrombina ou hiper-homocisteinemia & trombofilias isoladamente não modificam a \\
& conduta) \\
\hline
\end{tabular}

${ }^{a}$ Considerar o equilíbrio entre o risco de recorrência de TEV e a chance de sangramento. ${ }^{\text {b}}$ Trombofilias de alto risco: homozigose para fator $\mathrm{V}$ Leiden, mutação do gene da protrombina, deficiência de proteína $\mathrm{C}$, de proteína $\mathrm{S}$ ou de antitrombina e síndrome antifosfolipídeo. 
vista a reavaliação e a discussão sobre a reintrodução do tratamento.(B)

As recomendações para a duração do tempo de terapia anticoagulante estão sumarizadas no Quadro 12.

\section{Referências}

1. Kearon C. Duration of therapy for acute venous thromboembolism. Clin Chest Med. 2003;24(1):63-72.

2. Agnelli G, Prandoni P, Becattini C, Silingardi M, Taliani MR, Miccio M, et al. Extended oral anticoagulant therapy after a first episode of pulmonary embolism. Ann Intern Med. 2003;139(1):19-25.

3. Murin S, Romano PS, White RH. Comparison of outcomes after hospitalization for deep venous thrombosis or pulmonary embolism. Thromb Haemost. 2002;88(3):407-14.

4. Hull R, Delmore T, Genton E, Hirsh J, Gent M, Sackett $\mathrm{D}$, et al. Warfarin sodium versus low-dose heparin in the long-term treatment of venous thrombosis. N Engl J Med. 1979;301(16):855-8.

5. Ridker PM, Goldhaber SZ, Danielson E, Rosenberg Y, Eby CS, Deitcher SR, et al. Long-term, low-intensity warfarin therapy for the prevention of recurrent venous thromboembolism. N Engl J Med. 2003;348(15):142534.

6. Kearon C, Ginsberg JS, Kovacs MJ, Anderson DR, Wells P, Julian JA, et al. Comparison of low-intensity warfarin therapy with conventional-intensity warfarin therapy for long-term prevention of recurrent venous thromboembolism. N Engl J Med. 2003;349(7):631-9.

7. van der Heijden JF, Hutten BA, Büller HR, Prins MH. Vitamin $\mathrm{K}$ antagonists or low-molecular-weight heparin for the long term treatment of symptomatic venous thromboembolism. Cochrane Database Syst Rev. 2002;(1):CD002001.

8. lorio A, Guercini F, Pini M. Low-molecular-weight heparin for the long-term treatment of symptomatic venous thromboembolism: meta-analysis of the randomized comparisons with oral anticoagulants. J Thromb Haemost. 2003;1(9):1906-13.

9. Torbicki A, Perrier A, Konstantinides S, Agnelli G, Galiè $\mathrm{N}$, Pruszczyk P, et al. Guidelines on the diagnosis and management of acute pulmonary embolism: the Task Force for the Diagnosis and Management of Acute Pulmonary Embolism of the European Society of Cardiology (ESC). Eur Heart J. 2008;29(18):2276-315.

10. Ruíz-Giménez N, Suárez C, González R, Nieto JA, Todolí JA, Samperiz AL, et al. Predictive variables for major bleeding events in patients presenting with documented acute venous thromboembolism. Findings from the RIETE Registry. Thromb Haemost. 2008;100(1):26-31.

11. Schulman S, Rhedin AS, Lindmarker P, Carlsson A, Lärfars G, Nicol P, et al. A comparison of six weeks with six months of oral anticoagulant therapy after a first episode of venous thromboembolism. Duration of Anticoagulation Trial Study Group. N Engl J Med. 1995;332(25):1661-5.

12. Optimum duration of anticoagulation for deepvein thrombosis and pulmonary embolism. Research Committee of the British Thoracic Society. Lancet. 1992;340(8824):873-6.

13. Campbell IA, Bentley DP, Prescott RJ, Routledge PA, Shetty HG, Williamson 1J. Anticoagulation for three versus six months in patients with deep vein thrombosis or pulmonary embolism, or both: randomised trial. BMJ. 2007;334(7595):674.

14. Pinede L, Ninet J, Duhaut P, Chabaud S, DemolombeRague S, Durieu 1, et al. Comparison of 3 and 6 months of oral anticoagulant therapy after a first episode of proximal deep vein thrombosis or pulmonary embolism and comparison of 6 and 12 weeks of therapy after isolated calf deep vein thrombosis. Circulation. 2001;103(20):2453-60.

15. Hutten BA, Prins MH. Duration of treatment with vitamin $\mathrm{K}$ antagonists in symptomatic venous thromboembolism. Cochrane Database Syst Rev. 2006;(1):CD001367.

16. Ost D, Tepper J, Mihara H, Lander 0, Heinzer R, Fein A. Duration of anticoagulation following venous thromboembolism: a meta-analysis. JAMA. 2005;294(6):706-15.

17. Kearon C, Kahn SR, Agnelli G, Goldhaber S, Raskob GE, Comerota AJ, et al. Antithrombotic therapy for venous thromboembolic disease: American College of Chest Physicians Evidence-Based Clinical Practice Guidelines (8th Edition). Chest. 2008;133(6 Suppl):454S-545S.

18. Hutten BA, Prins MH, Gent M, Ginsberg J, Tijssen JG, Büller HR. Incidence of recurrent thromboembolic and bleeding complications among patients with venous thromboembolism in relation to both malignancy and achieved international normalized ratio: a retrospective analysis. J Clin Oncol. 2000;18(17):3078-83.

19. Lee AY, Levine MN, Baker Rl, Bowden C, Kakkar AK, Prins $\mathrm{M}$, et al. Low-molecular-weight heparin versus a coumarin for the prevention of recurrent venous thromboembolism in patients with cancer. $\mathrm{N}$ Engl J Med. 2003;349(2):146-53.

20. Akl EA, Barba M, Rohilla S, Terrenato 1, Sperati F, Muti $\mathrm{P}$, et al. Anticoagulation for the long term treatment of venous thromboembolism in patients with cancer. Cochrane Database Syst Rev. 2008;(2):CD006650.

21. Ho WK, Hankey GJ, Quinlan DJ, Eikelboom JW. Risk of recurrent venous thromboembolism in patients with common thrombophilia: a systematic review. Arch Intern Med. 2006;166(7):729-36.

22. Seligsohn U, Lubetsky A. Genetic susceptibility to venous thrombosis. N Engl J Med. 2001;344(16):1222-31.

23. Institute for Clinical Systems Improvement. Venous Thromboembolism Diagnosis and Treatment. Bloomington: Institute for Clinical System Improvement; 2009.

24. Eriksson Bl, Dahl OE, Rosencher N, Kurth AA, van Dijk CN, Frostick SP, et al. Oral dabigatran etexilate vs. subcutaneous enoxaparin for the prevention of venous thromboembolism after total knee replacement: the RE-MODEL randomized trial. J Thromb Haemost. 2007;5(11):2178-85.

25. Eriksson Bl, Dahl OE, Rosencher N, Kurth AA, van Dijk CN, Frostick SP, et al. Dabigatran etexilate versus enoxaparin for prevention of venous thromboembolism after total hip replacement: a randomised, double-blind, non-inferiority trial. Lancet. 2007;370(9591):949-56.

26. Capriani JA, Hwang E, Hantel S. The oral direct thrombin inhibitor, dabigatran etexilate is effective and sage for prevention of major venous thromboembolism following major orthopedic surgery [abstract]. J Thromb Haemost. 2007;5(Suppl 2):0-W-050.

27. Palareti G, Cosmi B, Legnani C, Tosetto A, Brusi $\mathrm{C}$, lorio $\mathrm{A}$, et al. D-dimer testing to determine the duration of anticoagulation therapy. $\mathrm{N}$ Engl J Med. 2006;355(17):1780-9. Erratum in: N Engl J Med. 2006;355(26):2797. 